\title{
Thick-target inverse kinematic method in order to investigate alpha-clustering in ${ }^{212} \mathrm{Po}$
}

\author{
Maria Grazia Pellegriti ${ }^{1, *}$, Agatino Musumarra ${ }^{1,2}$, Enrico De Filippo ${ }^{1}$, Marzio De Napoli ${ }^{1}$, Alessia Di Pietro ${ }^{3}$, \\ Pierpaolo Figuera ${ }^{3}$, Maria Fisichella ${ }^{3}$, Concettina Maiolino $^{3}$, Danilo Rifuggiato ${ }^{3}$, Domenico Santonocito ${ }^{3}$, \\ Valentina Scuderi ${ }^{3}$, Emanuele Strano ${ }^{4,5}$, Domenico Torresi ${ }^{3}$ \\ ${ }^{1}$ Istituto Nazionale di Fisica Nucleare - Sezione di Catania, via Santa Sofia n. 64, 95123 Catania, Italy \\ ${ }^{2}$ Dipartimento di Fisica ed Astronomia, Università di Catania, via Santa Sofia n.64, 95123 Catania, Italy \\ ${ }^{3}$ Istituto Nazionale di Fisica Nucleare - Laboratori Nazionali del Sud, via Santa Sofia n. 62, 95123 Catania, Italy \\ ${ }^{4}$ Dipartimento di Fisica, Università di Padova, via F. Marzolo 8, I-35131 Padova, Italy \\ ${ }^{5}$ Istituto Nazionale di Fisica Nucleare - Sezione di Padova, via F. Marzolo 8, I-35131 Padova, Italy
}

\begin{abstract}
The inverse-kinematic thick-target method has been used in order to investigate ${ }^{212} \mathrm{Po}$ alphastructure by the elastic scattering of ${ }^{208} \mathrm{~Pb}$ on ${ }^{4} \mathrm{He}$ target. A ${ }^{208} \mathrm{~Pb}$ beam, accelerated by the Superconducting Cyclotron (CS) of Laboratori Nazionali del Sud - INFN, at the incident energy of $10.1 \mathrm{~A} \mathrm{MeV} \mathrm{was}$ impinging onto a specifically designed ${ }^{4} \mathrm{He}$ gas cell, two meter long. The gas cell was acting both as target and as beam degrader, stopping the beam before reaching the alpha-particle detection system placed at $0^{\circ}$ with respect to the beam axis. In order to disentangle the elastic contribution from other reaction channels (e.g. inelastic scattering) a microchannel plate was used to measure the Time of Flight (ToF) of both the ${ }^{208} \mathrm{~Pb}$ beam particles and the ejectiles along the gas cell. The ${ }^{208} \mathrm{~Pb}$ stopping power in the ${ }^{4} \mathrm{He}$ gas target was also measured, as a key ingredient in order to establish the interaction point inside the gas cell, in turn determining the solid angle covered by the detector. In the following, the experimental technique will be described, and the results of a preliminary data analysis will be shown.
\end{abstract}

\section{Introduction}

The $\alpha$-particle is considered as one of the basic building blocks of nuclei because of its large binding energy [1]. Opposite to this picture is the single particle description of nuclei, based on the hypothesis of a common mean field (shell model). A strong revival of the $\alpha$-cluster model occurred in the 1960s when both experimental and theoretical studies revealed that the concept of $\alpha$ clustering is essential for understanding the structure of light nuclei. Nuclear states based on $\alpha$ clusters have been mainly observed as excited states, close to the decay thresholds into the constituent clusters [1].

The persistence of clustering in heavy-nuclei is much less documented. The interesting question is whether the alpha cluster structure can survive in a mean field approach. It is traditionally believed that the mean field dominated in heavy nuclei. For medium nuclei, it was found in $[2,3]$ that there exist the inversion doublet bands in the fp-shell ${ }^{40} \mathrm{Ca}$ and ${ }^{44} \mathrm{Ti}$, thus supporting the hypothesis they could have an $\alpha+$ core cluster structure, like the well-known structure of the light ${ }^{16} \mathrm{O}$ and ${ }^{20} \mathrm{Ne}$ nuclei. Furthermore, a heavy closed-shell core plus alpha can also be described in terms of a cluster structure. In this framework, a possible candidate is the ${ }^{208} \mathrm{~Pb}+\alpha$ structure characterizing ${ }^{212} \mathrm{Po}$ [4], a nucleus exhibiting two protons and two neutrons outside the doubly magic ${ }^{208} \mathrm{~Pb}$ core. The enhancement of the $\alpha$-decay width in ${ }^{212} \mathrm{Po}$ was studied in [5] and it was found that the larger configuration mixing plays a very important role in the explanation of the width enhancement.

New interest, from both experimental [6] and theoretical point of view [7-9], raised around ${ }^{212}$ Po and recently a review on alpha-clustering effects in heavy nuclei has been published [10].

In the work of Astier et al. [6], the discovery of lowexcited negative parity states in ${ }^{212} \mathrm{Po}$ is claimed in the $\mathrm{E}_{\mathrm{x}}$ region below $3 \mathrm{MeV}$, with evidence of very enhanced $\mathrm{E}_{1}$ transitions. According to [6], these states can e considered as fingerprints of the $\alpha-{ }^{208} \mathrm{~Pb}$ structure, a signature of the $\alpha$-core oscillatory motion. Later Suzuki et al. [7] has claimed that the experimental evidence of [6] has to be attributed to the strong contribution of an $\alpha$ ${ }^{208} \mathrm{~Pb}\left(3^{-}, 2.615 \mathrm{MeV}\right)$ cluster state.

In order to further investigate ${ }^{212} \mathrm{Po} \alpha$-structure, the elastic scattering ${ }^{4} \mathrm{He}\left({ }^{208} \mathrm{~Pb}, \alpha\right){ }^{208} \mathrm{~Pb}$ has been measured at Laboratori Nazionali del Sud - INFN by using the thick-target inverse kinematics (TTIK) resonant elastic scattering method in the $E_{c m}$ energy range below 30 $\mathrm{MeV}$.

\footnotetext{
* Corresponding author: mariagrazia.pellegriti@ct.infn.it
} 


\section{The method}

The TTIK resonant elastic scattering method [11-14] makes use of the sensitivity of the detected recoil yield to the presence of a resonant state in the compound nucleus. By using the beam energy loss in the target, it is possible to continuously induce the elastic scattering in a given range of $\mathrm{E}_{\mathrm{CM}}$ energies. The inverse kinematics scattering (projectile heavier than the target nucleus), produces forward focused recoils (typically protons or $\alpha$ particles) with much smaller energy loss in the target than the beam particles. The detected recoil yield shows interference effects due to the presence of both nuclear and Coulomb contribution in the elastic cross section. The choice of a proper target thickness is essential for implementing this method and it will provide in one step a general overview of the level scheme in a wide centreof-mass energy range.

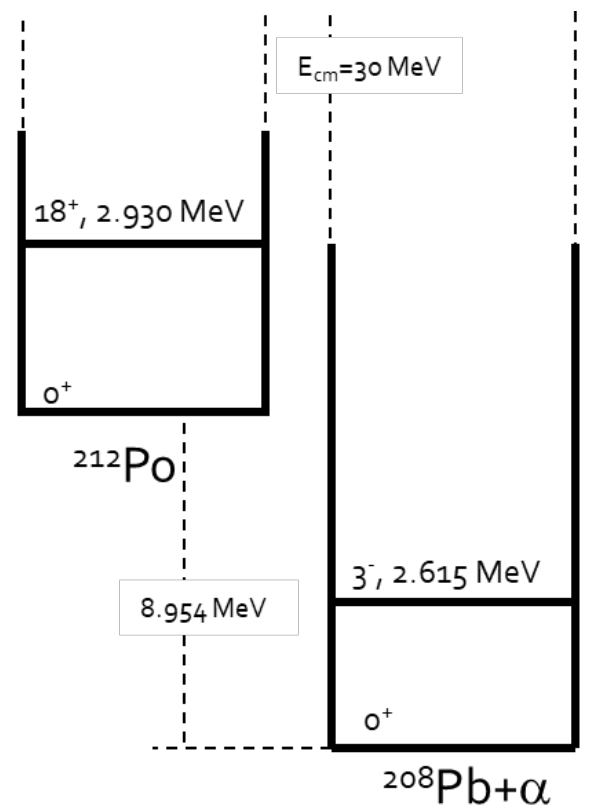

Fig. 1. ${ }^{208} \mathrm{~Pb}$ and ${ }^{212} \mathrm{Po}$ level scheme.
The experimental limitation of the thick target method is represented by the contamination, coming from other possible reaction mechanisms (like inelastic scattering), of the final elastic scattering excitation function.

This means that recoil particles reaching the detectors at the same energy can be generated by different reaction mechanisms. This implies different paths inside the target for different classes of events. In order to experimentally disentangle the elastic cross section excitation function, an appropriate target thickness is provided [13] or, as a further option for gaseous target, ToF measurement is performed [14].

As already stated, we have investigated the ${ }^{212} \mathrm{Po} \alpha-{ }^{208} \mathrm{~Pb}$ structure around the Coulomb barrier by using the TTIK; Fig. 1 reports a simplified scheme of the involved levels configuration in ${ }^{212} \mathrm{Po}$ and ${ }^{208} \mathrm{~Pb}$ nuclei and the investigated $\mathrm{E}_{\mathrm{cm}}$ energy range.

As it has been demonstrated in [14], the knowledge of target stopping power for the traversing beam is crucial in performing elastic scattering experiments by using the thick target method.

For this reason, a measurement of ${ }^{4} \mathrm{He}$ stopping power for a ${ }^{208} \mathrm{~Pb}$ beam in the energy range spanning from $1.588 \mathrm{GeV}$ down to zero has been performed.

\section{Set-up}

The ${ }^{208} \mathrm{~Pb}$ beam $\left(31^{+}\right.$charge state), extracted from the SERSE ECR source, has been accelerated by the CS at the beam energy of $10.1 \mathrm{~A} \cdot \mathrm{MeV} .{ }^{208} \mathrm{~Pb}$ particles were impinging on a Micro Channel Plate (MCP) detector. The MCP foil has been placed at $45^{\circ}$ with respect to the beam direction. The MCP signal provides a reference for time-of-flight measurements and, at the same time, allows beam particle counting.

The beam was then impinging on the ${ }^{4} \mathrm{He}$ gas target (see Fig. 2 and Fig. 3) separated by a beam-line by an Al window, $20 \mu \mathrm{m}$ thick (see Fig. 4). The gas target length, from the Al window to the detector, placed at the end of the gas target (see Fig. 2), was $1834 \pm 1 \mathrm{~mm}$. The ${ }^{4} \mathrm{He}$ gas pressure inside the beam pipe was measured by using an absolute pressure gauge (MKS Baratron 122A). The temperature was also measured by using a Pt100 probe.

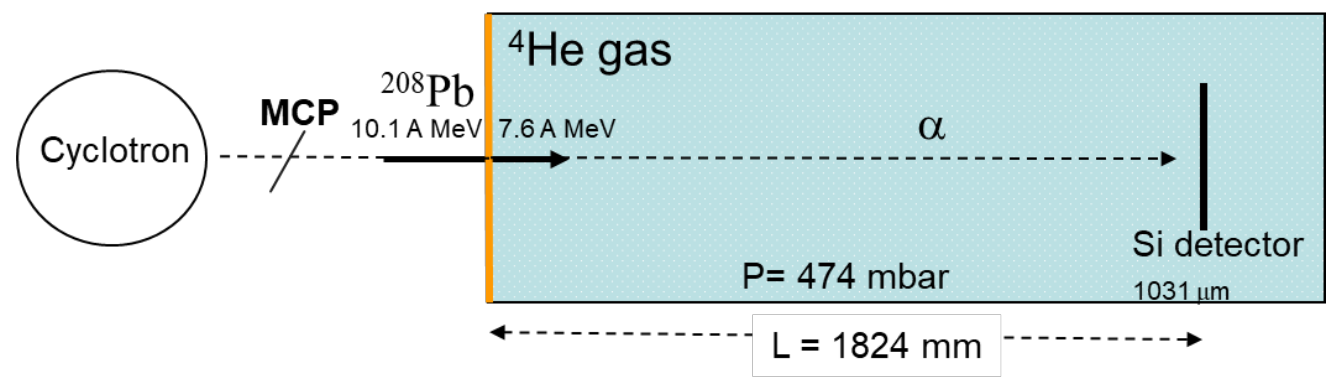

Fig. 2. Experimental set-up. 


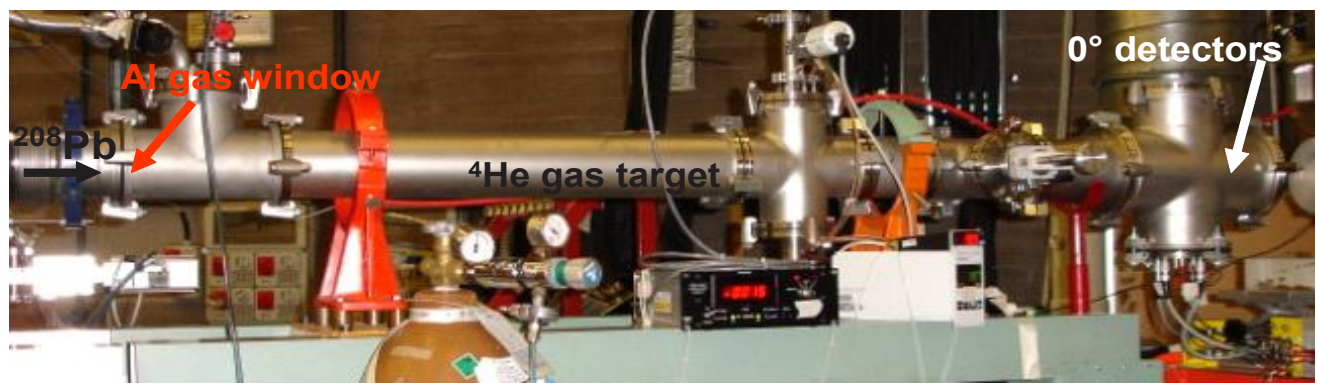

Fig. 3. Picture of ${ }^{4} \mathrm{He}$ gas chamber.

During the experiment two different detector set-ups were used. In the following we will refer to the result obtained by the set-up based on an ORTEC silicon surface-barrier detector, $1031 \mu \mathrm{m}$ thick, placed at zero degree detecting the recoiling alpha-particles.

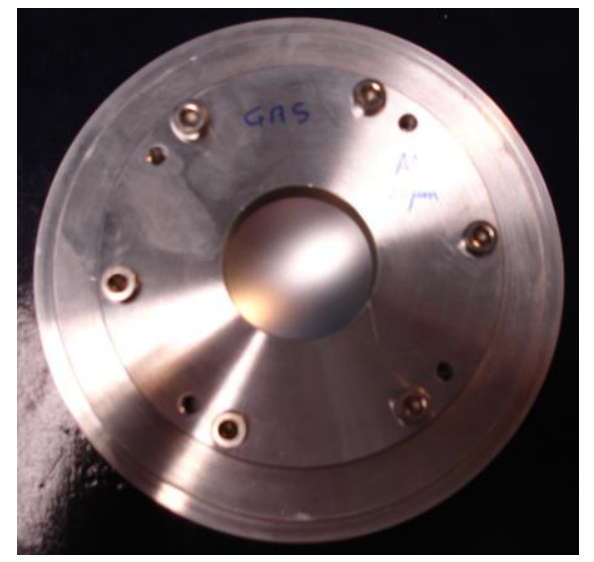

Fig. 4. Al gas window.

The detector was collimated $(\phi=6 \mathrm{~mm})$ and used as start detector (trigger) for ToF measurement, stop was provided by the MCP detecting the primary ${ }^{208} \mathrm{~Pb}$ beam particles. Overall ToF time resolution has been measured to be $\sim 1 \mathrm{~ns}$.

Stopping power measurements of ${ }^{208} \mathrm{~Pb}$ in ${ }^{4} \mathrm{He}$ have also been performed. As it has been underlined by Zadro et al. [14], this is essential in order to recover the beam energy for a given scattering event.

\section{Preliminary data analysis}

The detector energy calibration was performed by using an ${ }^{241} \mathrm{Am}-{ }^{244} \mathrm{Cu}-{ }^{239} \mathrm{Pu}$ alpha-source, the energy corresponding to the alpha particles punching through the silicon detector and the ADC pedestal. The ToF was calibrated by using different delay, namely: 8, 16 and 24 ns.

ToF versus detected energy $2 \mathrm{D}$-spectrum is plotted in Fig. 5. Elastic scattered alpha particles appear as the main contribution.

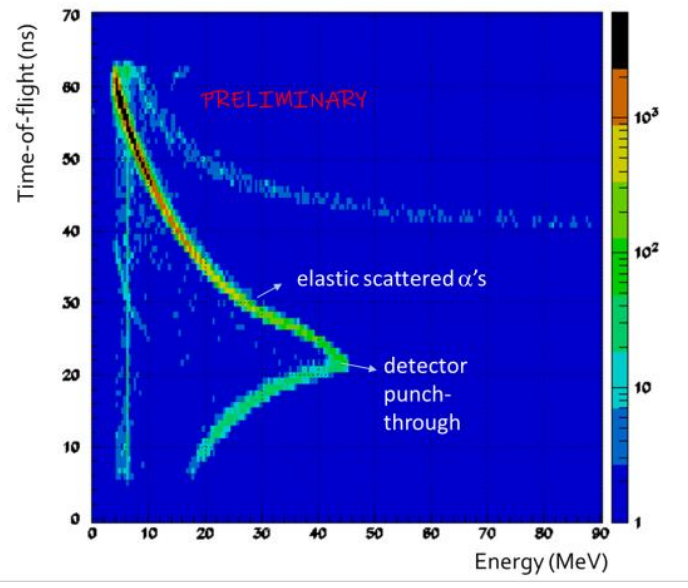

Fig. 5. Time-of-flight vs detected energy $2 \mathrm{D}$-spectrum. The elastic scattering events in the lower part refer to alpha particles scattered close to the entrance Al-window.

These are clearly disentangled from a contamination related to heavier ejectiles produced in the ${ }^{27} \mathrm{Al}$ window (upper part) and protons (left side). The vertical, timeuncorrelated line, has been generated by alpha particles produced by the decay of heavy residues implanted in the Al-window. A full GEANT-4 simulation is in progress in order to take into account the setup geometry and all the possible sources of contamination.

A preliminary data analysis has been performed for the low energy alpha particles which stop in the detector. For these events the centre-of-mass energy have been recovered and, consequently, the cross-section deduced. A function correlating centre of mass energy vs lab energy (alpha's detected energy) has been calculated by using the LISE++ [15] tools. It has been used during the analysis in order to convert the detected alpha energy into centre-of-mass energy. At the same time the detector solid angle as a function of the centre-of-mass energy has been calculated in order to obtain the elastic cross-section. Finally, the experimental cross-section has been normalized to the Rutherford cross section at $\Theta_{\mathrm{cm}}=180^{\circ}$, thus to obtain the absolute cross section shown in Fig. 6. 


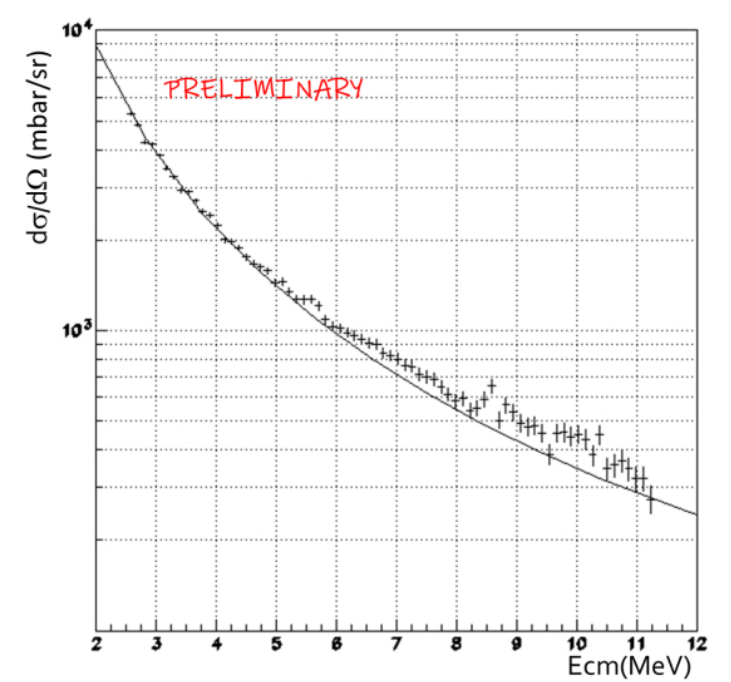

Fig. 6. Experimental centre-of-mass cross-section distribution. Absolute cross section was obtained by normalizing experimental low energy data to Rutherford cross section (solid line).

As shown in Fig. 6, the experimental trend agrees with the Rutherford cross section at low energy, but in the energy region starting from $8 \mathrm{MeV}$, the experimental cross section exceeds the theoretical one. Must be underlined that the Coulomb-barrier height of the ${ }^{208} \mathrm{~Pb}$ $\alpha$ system is $26 \mathrm{MeV}$ (classical Coulomb barrier), thus it is striking to have evidence of structures at such low $\mathrm{E}_{\mathrm{cm}}$ energies. Results from [16,17], obtained by using an effective alpha-particle structure model, where the particle is bound to a doubly magic nucleus, show that $\alpha$ clustering can occur near the surface of the heavy core. This means, the $\alpha$-like clustering can be found in nuclear system at a low density region. Furthermore, Tohsaki and Itagaki $[18,19]$ assume that some heavy nuclei can have specific $\alpha$-clustering geometrical structure where the $\alpha$ cluster is considered as the building block. These geometric configurations can be favoured in very heavy (super-heavy nuclei) when $\alpha$ clusters form a nucleus and the Coulomb repulsion, most important at large distances, is strongly suppressed by forming the hollow structures [18].

\section{Conclusions}

In order to investigate ${ }^{212} \mathrm{Po}$ alpha-structure, the thicktarget inverse kinematic method has been used in order to study elastic scattering of ${ }^{208} \mathrm{~Pb}$ on ${ }^{4} \mathrm{He}$ target. The preliminary data analysis has shown an interesting deviation of the elastic cross-section at energies well below the Coulomb barrier. Such behaviour can be explained in the framework of a strong $\alpha$-clustering in ${ }^{212}$ Po. A full simulation of the experimental set-up and a further data analysis, on data obtained during the same experiment by using a different detector set-up, will be performed in order to confirm such a preliminary evidence.

\section{References}

1. K. Ikeda, T. Marumori, R. Tamagaki, H. Tanaka Suppl. Progr. Theor. Phys. 52, 1 (1972)

2. T. Tamaya, K. Katory, M. Fujiwara, S. Kato and S. Ohkhubo Prog. Theor. Phys. 132, 73 (1998)

3. T. Sakuda and S. Ohkubo Prog. Theor. Phys. 132, 103 (1998)

4. R. G. Lovas, R. J. Liotta, A. Insolia, K. Varga and D. S. Dellion Phys. Rep. 294, 265 (1998)

5. I. Tonozuka and A. Arima Nucl. Phys. A323, 45 (1979)

6. A. Astier, P. Petkov, M.-G. Porquet, D.S. Delion and P. Schuck Phys. Rev. Lett. 104, 0427701 (2010)

7. Y. Suzuki and S. Ohkubo Phys. Rev. C 82, 041303(R) (2010)

8. T.T. Ibrahim, S. M. Perez, and S. M. Wyngaardt Phys. Rev. C 82, 034302 (2010)

9. Dongdong Ni and Zhongzhou Ren Phys. Rev. C 83, 014310 (2011)

10. Zhongzhou Ren and Bo Zhou Front. Phys. 13(6) 132110 (2018)

11. V. Z. Goldberg, G. V. Rogachev, W. H. Trzaska, J. J. Kolata, A. Andreyev, C. Angulo, M. J. G. Borge, S. Cherubini, G. Chubarian, G. Crowley, P. Van Duppen, M. Gorska, M. Gulino, M. Huyse, P. Jesinger, K.-M. Källman, M. Lattuada, T. Lönnroth, M. Mutterer, R. Raabe, S. Romano, M. V. Rozhkov, B. B. Skorodumov, C. Spitaleri, O. Tengblad, and A. Tumino, Phys. Rev. C 69, 024602 (2004)

12. C. Angulo, G. Tabacaru, M. Couder, M. Gaelens, P. Leleux, A. Ninane, F. Vanderbist, T. Davinson, P. J. Woods, J. S. Schweitzer, N. L. Achouri, J. C. Angélique, E. Berthoumieux, F. de Oliveira Santos, P. Himpe, and P. Descouvemont Phys. Rev. C 67, 014308 (2003)

13. M. G. Pellegriti, N.L. Achouri, C.Angulo, J.-C. Angélique, E.Berthoumieux, E. Casarejos, M. Couder, T. Davinson, C. Ghag, A. St. Murphy N. A. Orr, I. Raye, I. G. Stefane, P. Descouvemont Phys. Lett. B 659 (2008) 864

14. M. Zadro, A. Di Pietro, P. Figuera, M. Fisichella, M. Lattuada, A. Maggio, F. Pansini, M. Papa, V. Scuderi, O.Yu. Goryunov, V.V. Ostashko Nucl. Instr. Meth. B259, 836 (2007)

15. LISE++, http://lise.nscl.msu.edu/lise.html

16. G. Röpke, P. Schuck, Y. Funaki, H. Horiuchi, et al., Phys. Rev. C 90, 034304 (2014)

17. C. Xu, Z. Ren, G. Röpke, P. Schuck, et al., Phys. Rev. C 93, 011306 (2016)

18. A. Tohsaki and N. Itagaki, Phys. Rev. C 97, 011301 (2018)

19. A. Tohsaki and N. Itagaki, Phys. Rev. C 98, 014302 (2018) 